Yunwang Li

Sumei Dai $ه$

Feng Tian

Yuwei Zheng

https://doi.org/10.21278/TOF.43Si102

ISSN 1333-1124

eISSN 1849-1391

\title{
STAIRS-CLIMBING CAPACITY OF A W-SHAPED TRACK ROBOT
}

\author{
Summary
}

Stairs-climbing capacity is an important index for obstacle-overcoming performance for coal mine rescue robot. For studying on stairs-climbing capacity of the coal mine rescue robot with W-shaped track suspensions, theoretical analysis, kinematics simulations and tests on real stairs have been carried out. According to sizes of normal stairs in reality, there are two climbing situations: (1) the first track section and the third track section climb the nosings of the two adjacent steps or two septal steps successively, (2) the front and rear tracks climb two nosings simultaneously. There are two critical states: (1) the first and third track section just touch the nosing of two interval steps, (2) the first and third track section barely touches the nosings of the adjacent steps. According to the two states, equation sets which describe the relationship between tread depth and riser height are deduced. According to the physical dimensions of the robot, the relation curves are drawn corresponding to the equation sets. Conditions of the two climbing situations are obtained. The stairs-climbing are simulated on RecurDyn, and tests on a simulated staircase test platform in laboratory and on real stairs in and outside the building are carried out. The simulation and tests results are in accordance with the theoretical analysis. Research in this paper is conducive to optimizing the shape and size of the W-shaped track suspension according to the parameters of the stairs, and also contributes to guiding the application of the robot with a $\mathrm{W}$-shaped track moving mechanisms.

Key words: $\quad W$-shaped track robot; stairs-climbing capacity; kinematics simulation; continuous stairs-climbing test

\section{Introduction}

After gas and coal dust explosion accidents in underground coal mine, rescue mission is extremely dangerous. In order to perform the rescue mission successfully and quickly, it is necessary to send coal mine rescue robots to enter the mine disaster area and detect environment after the disaster $[1,2]$. The primary task of the rescue work for the robots is to enter the disaster area of underground coal mine. Terrain in underground coal mine after explosion disaster is unstructured [3]. There are artificially built independent steps, continuous stairs, slopes, drainage channels and rails in the laneways. Therefore, we should focus on the moving mechanism which is a difficulty in research field of the coal mine rescue robot. Sometimes, the coal mine robots have to try to climb continuous stairs. Therefore, the 
stairs-climbing capacity of the robot is an important index to evaluate the terrain passing performances. In order to obtain good performance of climbing stairs, some mine coal robots adopt joint track arms $[4,5]$ or special shape tracks such as inverted trapezoidal track [6] and triangular track. The robot with rocker type $\mathrm{W}$-shaped track moving mechanism has the advantages of mobile performance of wheeled robot and tracked robot, and has a good ability to adapt to rough ground and climb over structural obstacles [7]. This paper introduces a rocker type asymmetrical W-shaped track robot [8-10], which has two asymmetrical Wshaped track suspension linked on the both side of the main body. In order to study on the stairs-climbing capacity of the W-shaped track moving mechanism, theoretical analysis, kinematics simulation and tests on real stairs have been carried out. This work contributes to the shape and size optimization and application of the $\mathrm{W}$-shaped track robot.

\section{The rocker-type W-shaped track robot}

Figure 1 shows the mechanical structure of the W-shaped track robot, described in [10]. The platform is comprised of two asymmetrical W-shaped rocker track suspensions, a differential mechanism and a main body. The two track suspensions are hinged coaxially with the main body. Each suspension is comprised of a front and a rear track assemblies which are connected by chain transmission, and there is a tensioner idle outside each track of each track assembly. The track suspensions are driven by DC motors installed in the main body. The main body and the two suspensions are linked by the link rods type differential mechanism. The suspensions will rock with the rough terrain during the robot running in the unstructured environment. In structured terrain, track suspensions and main body can be considered fixed connection. The two track assemblies sections of each suspension are named front track section and rear track section, and the wheels of the track suspensions are signed as wheel 1 , 2, 3, 4 and 5, as shown in Figure 1. The robot's physical dimensions are marked in Figure 2 and listed in the Table 1.

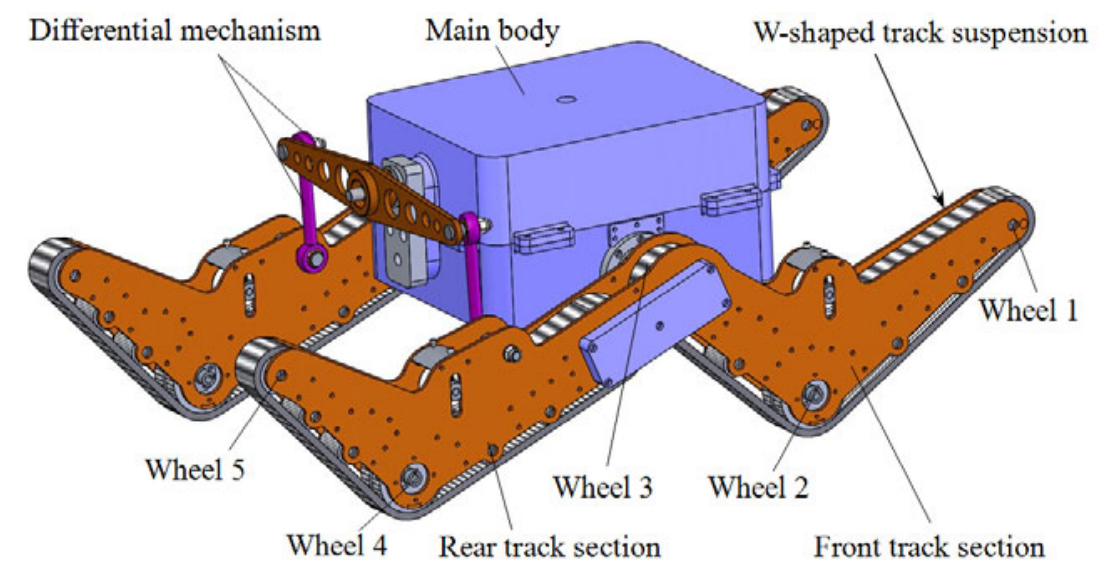

Fig. 1 Mechanical structure of the W-shaped track robot

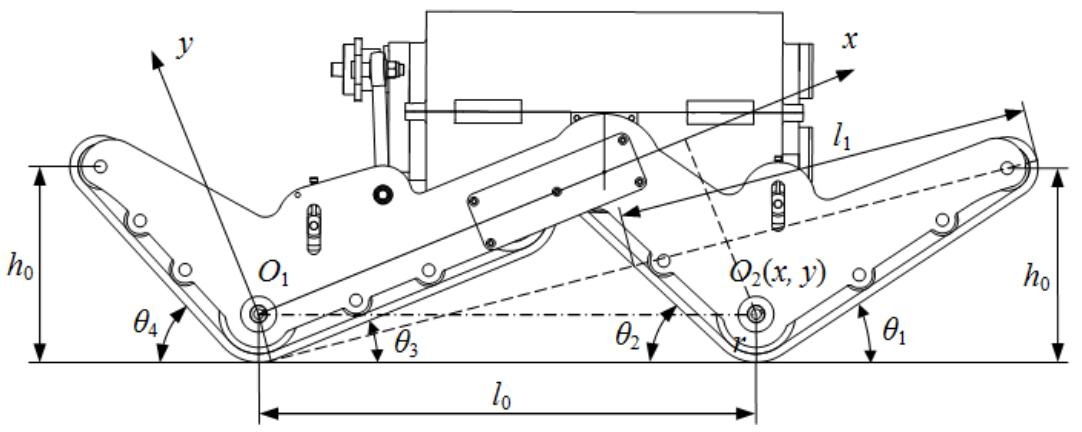

Fig. 2 Physical dimensions of the robot 


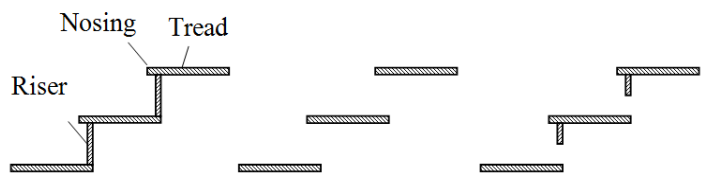

(a) (b)

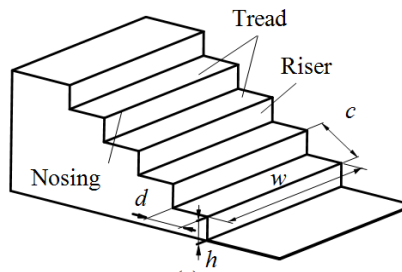

(a)

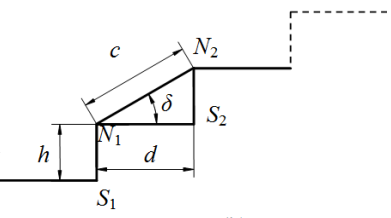

(b)

Fig. 3 Kinds of the stairs

Fig. 4 Simplification of the stairs

Table 1 Physical dimensions of the robot

\begin{tabular}{|c|c|c|c|c|c|c|c|c|}
\hline $\begin{array}{c}\text { Item } \\
\text { names }\end{array}$ & $\begin{array}{c}\text { Radius of } \\
\text { the } \\
\text { touchdown } \\
\text { wheel } \\
\text { (including } \\
\text { the track } \\
\text { thickness) }\end{array}$ & $\begin{array}{c}\text { Lifting } \\
\text { height of } \\
\text { the tracks }\end{array}$ & $\begin{array}{c}\text { Track } \\
\text { wheels } \\
\text { touchdown } \\
\text { length }\end{array}$ & $\begin{array}{c}\text { Length of a } \\
\text { transversal } \\
\text { serif }\end{array}$ & $\begin{array}{c}\text { Initial } \\
\text { elevation } \\
\text { of } \\
\text { track 3 }\end{array}$ & $\begin{array}{c}\text { Initial } \\
\text { elevation } \\
\text { of } \\
\text { track 1 }\end{array}$ & $\begin{array}{c}\text { Coordinate } \\
\text { values of } \\
\text { wheel 3 } \\
\text { centre O2 in } \\
\text { Figure 2 }\end{array}$ & $\begin{array}{c}\text { Gravity } \\
\text { centre } \\
\text { coordinates } \\
\text { (can be } \\
\text { adjusted) }\end{array}$ \\
\hline Signs & $r / \mathrm{mm}$ & $h_{0} / \mathrm{mm}$ & $l_{0} / \mathrm{mm}$ & $l_{1} / \mathrm{mm}$ & $\theta_{1} /{ }^{\circ}$ & $\theta_{3} /{ }^{\circ}$ & $O_{2}(x, y) / \mathrm{mm}$ & $G(x, y) / \mathrm{mm}$ \\
\hline $\begin{array}{c}\text { Numerical } \\
\text { values }\end{array}$ & 58 & 240 & 600 & 500 & 33.4 & 22.2 & $\begin{array}{c}(554.8,- \\
226.6)\end{array}$ & $(480,24.8)$ \\
\hline
\end{tabular}

\section{Kinds and simplification of stairs and steps}

Isolated steps and continuous stairs are most common in step terrains, and the structures and dimensions of steps or stairs in various locations are different. The common kinds of straight stairs are shown in Figure 3. According to the forms of risers, stairs are classified into closed riser stairs, open riser stairs and semi-closed riser stairs, which are shown respectively in Figure 3-(a), (b), and (c). The stairs are composed of tread and rises, the part of tread extending riser is called flange. Usually, edge of tread and riser is also called nosing. According to forms of riser, stairs are classified into closed riser stairs, open riser stairs and semi-closed riser stairs, which are shown as Figure 3. The common successive straight stairs can be simplified as vertical closed riser stairs, shown in Figure 4. There are three main parameters to describe characteristics of stairs: riser height $h$, tread depth $d$ and tread width $w$ [5]. The width $w$ decides whether the robot can pass the stair in width, and $h$ and $d$ are the primary parameters of stair-climbing performance.

\section{Continuous stairs climbing performances}

\subsection{Analysis on the characteristic of stairs-climbing}

The continuous stairs can be considered to be composed of a series of isolated steps. The continuous stairs-climbing process is different due to the different riser height and treads depth. The riser height and tread depth of the normal stairs is usually small than $250 \mathrm{~mm}$ and $500 \mathrm{~mm}$ respectively. If riser height and tread depth for the robot is very small in a certain special place, the steps just like a speed pump on slop road, it is not hard to climb for the robot, and the process of climbing is just like slope climbing. According to the sizes of normal stairs in or outside building, there are two climbing situations. 


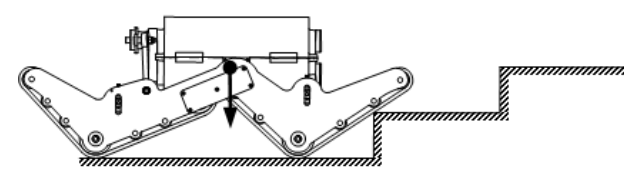

(a)
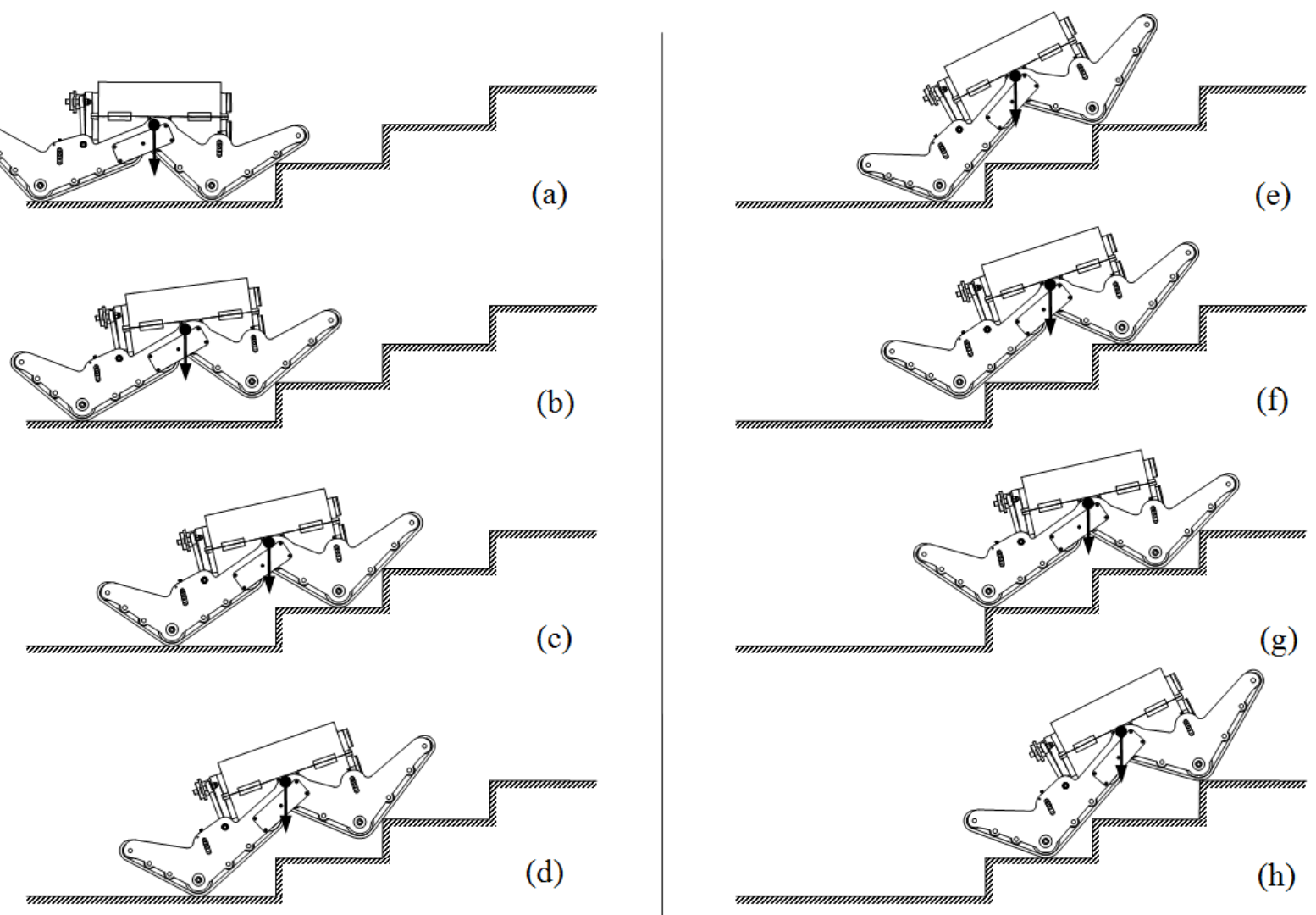

Fig. 5 Analysis of the first stairs-climbing situation
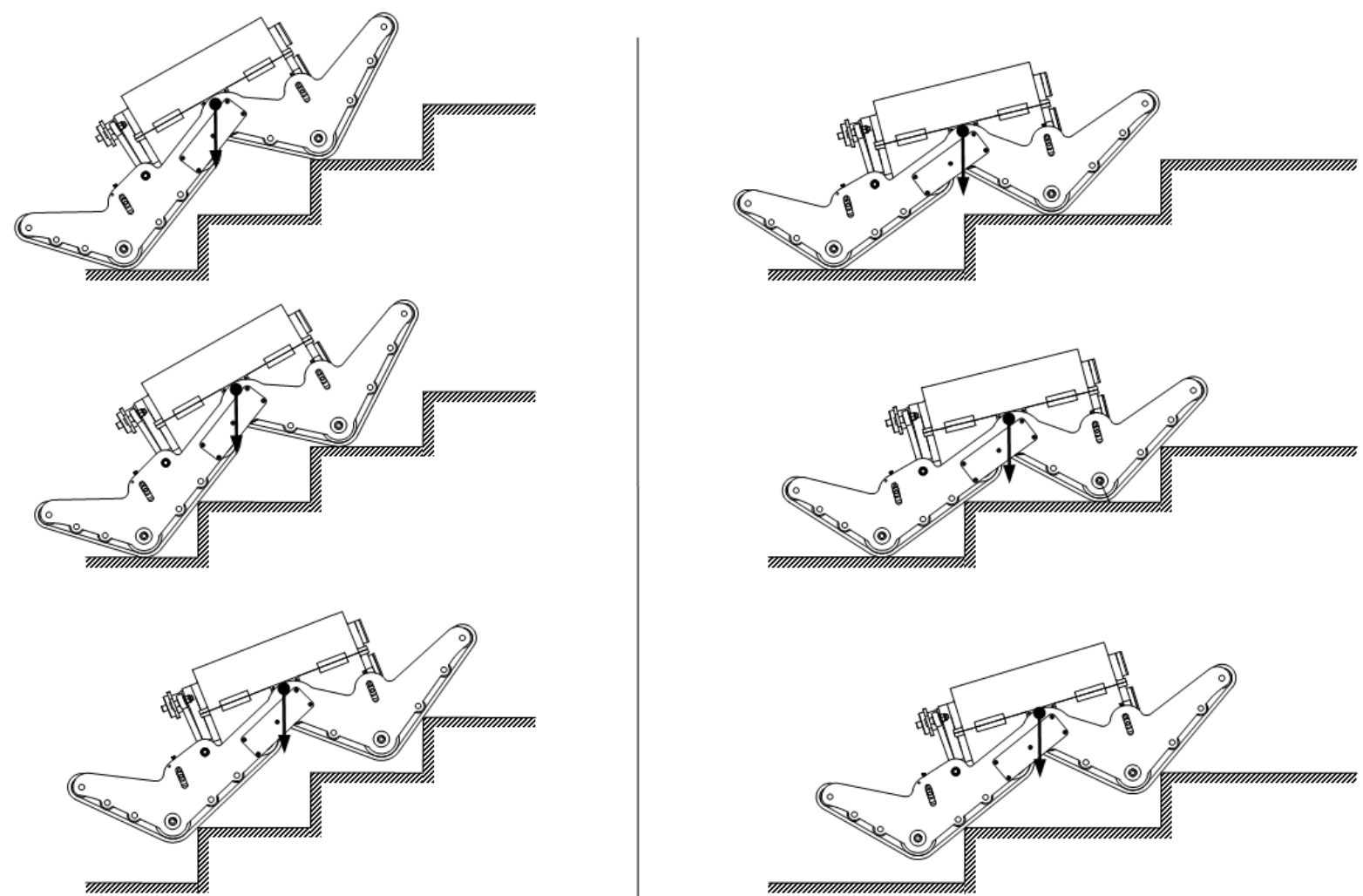

(a)

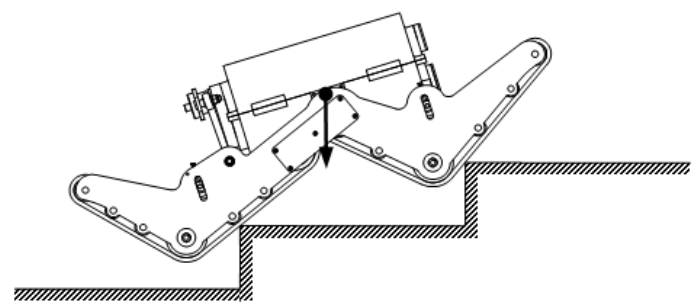

(b)

Fig. 6 Analysis of the second stairs-climbing situation 


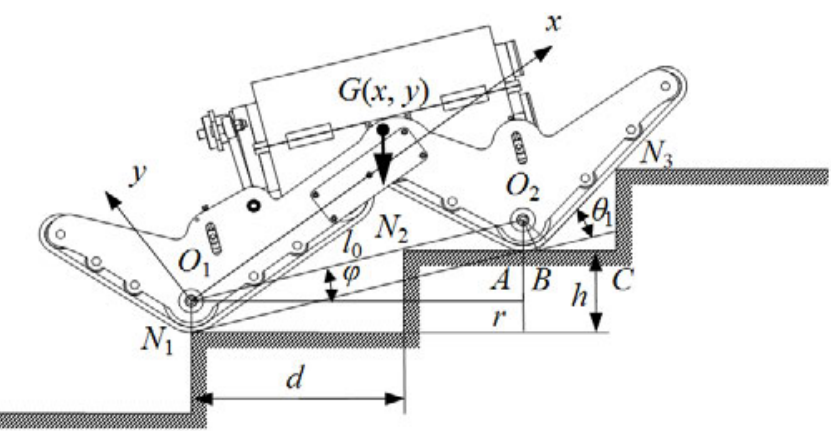

(a)

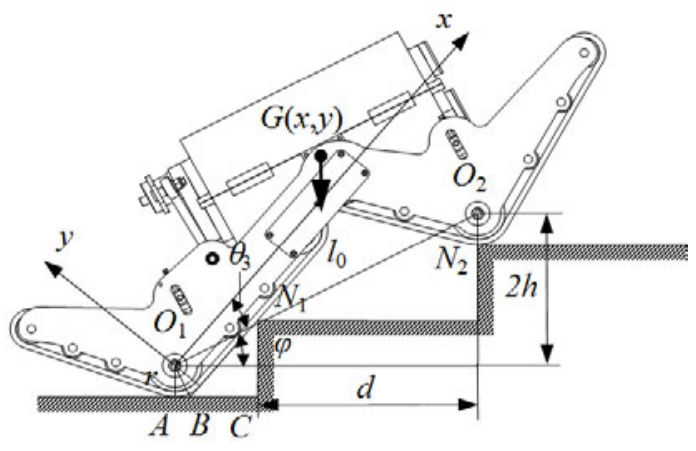

(b)

Fig. 7 Two critical states

The first situation is that the first track section and the third track section climb up the nosings of the two adjacent steps or two septal steps one by one. The climbing process of first situation is shown in Figure 5. When the front tracks of the robot just climb up one step's nosing, the rear tracks do not contact or just contact the nosing of the lower step, shown in Figure 5-(a-e). With the robot climbing upward, the rear tracks begin to climb the nosing of the lower step, shown in Figure 5-(f). When the rear tracks just have climbed up the lower step, its first track section still do not contact or just contact the nosing of the higher step, and the third section tracks do not contact the middle step's nosing, shown in Figure 5-(g). In this way, the robot have climbed on the first step, and been ready to climb the second one, shown in Figure 5-(h). In this situation, the robot smoothly and stably climbs on the steps of the stairs one by one.

The second situation is that the front and rear tracks climb two nosings simultaneously, as shown in Figure 6. In Figure 6-(a), the front and rear tracks climb the nosing of two interval steps. In Figure 6-(b), the front and rear tracks climb the nosing of two neighboring steps. In this situation, the first and third inclined tracks sections contact and climb the steps' nosings at the same time, only four points contact to the nosings of the stairs, if the friction coefficient is not high, the robot maybe slip along the slop direction.

The Provided horizontal projection of the gravity center of the robot is always before the contacting point in the horizontal direction; the robot can keep stable, and will not roll over backward. Although the gravity center may be changed in certain scope with the load and equipments changing, the special W-shaped track design can make the horizontal projection of the centroid at a safe position.

The two critical states are shown in Figure 7. In Figure 7-(a) the first and third track section just touches the nosing of two interval steps. In Figure 7-(b), the two track section barely touches the nosings of adjacent steps. And the two critical states satisfy the two equation sets (1) and (2).

According to the physical dimensions of the robot in Table 1, the relation curves $\mathrm{C} 1$ and $\mathrm{C} 2$ between the tread depth $\mathrm{d}$ and riser height $\mathrm{h}$ corresponding to the equation sets (1) and (2) respectively are shown in Figure 8, and the two curves intersect at a point $(153,371.5)$. At this point, the riser height $h=153 \mathrm{~mm}$, the tread depth $d$ is about $371.5 \mathrm{~mm}$. If the $h$ and $d$ are assigned values in region $A$ and $C$, the robot can climb the stairs using the first situation. If in region $B$ and $D$, the robot climbs the stairs according to the second situation.

$$
\left\{\begin{array}{l}
l_{0} \cdot \sin \varphi=h \\
l_{0} \cdot \cos \varphi+h \cdot \cot \left(\varphi+\theta_{1}\right)+r \cdot \tan \frac{\varphi+\theta_{1}}{2}=2 d
\end{array}\right.
$$




$$
\left\{\begin{array}{l}
l_{0} \cdot \sin \varphi=2 h \\
d+h \cdot \cot \left(\varphi+\theta_{3}\right)+r \cdot \tan \frac{\varphi+\theta_{3}}{2}=l_{0} \cdot \cos \varphi
\end{array}\right.
$$

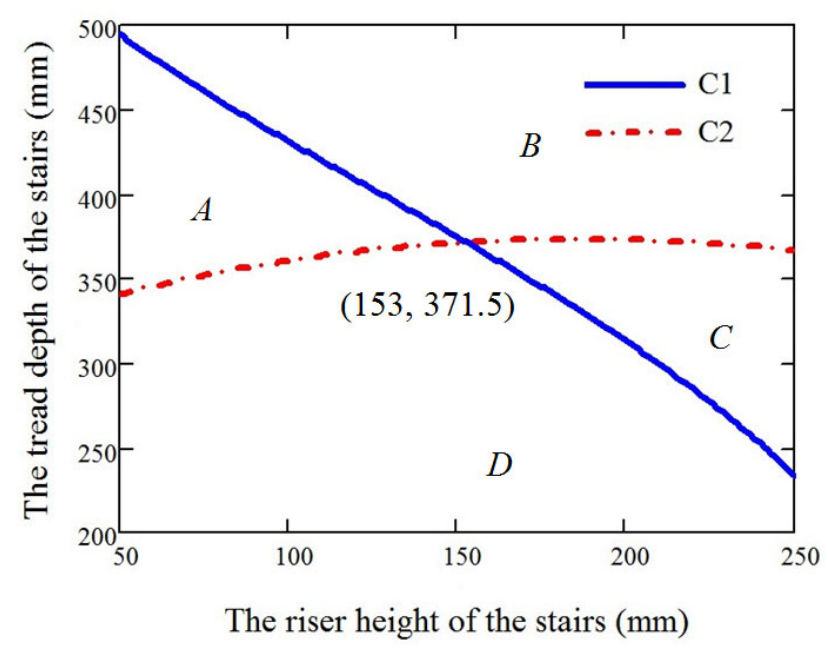

Fig. 8 Relation curves between tread depth and riser height

Take an example for analyzing. If the riser height $h$ is given, and $h$ is smaller than $153 \mathrm{~mm}$, a minim tread depth $d_{\min }(h)$ can be obtained according to the equation set (1), a maximum tread depth $d_{\max }(h)$ can be obtained according to the equation set (2). If the tread depth of one stairs is smaller than the minim tread depth $d_{\min }(h)$, the robot will climb the stairs with the first track and the third track touching the nosing of two interval steps simultaneously. If the tread depth of one stairs is larger than the maximum tread depth $d_{\max }(h)$, the robot will climb the stairs with the first and third tracks touching the nosing of two neighboring steps at the same time. If the tread depth $d \subset\left(d_{\min }(h), d_{\max }(h)\right)$, the robot will climb the stairs according to the first situation.

\subsection{Continuous stairs-climbing simulation}

A 3D model of the robot is imported into RecurDyn software [11]. A series of virtual stairs with $135 \mathrm{~mm}$ high-riser and $390 \mathrm{~mm}$ deep-thread are established in RecurDyn on the base of a series actual stairs outside a building. Additional series of virtual stairs with $150 \mathrm{~mm}$ high-riser and $270 \mathrm{~mm}$ deep-thread are also established. In the simulation system, the Y coordinate direction is vertical direction.

According to equation sets (1) and (2), when the riser height is $135 \mathrm{~mm}$, if the tread depth $d \subset(369,392)$, the robot's first and third track sections can climb the nosing in turn. The tread depth of the stairs is $390 \mathrm{~mm}$, so the robot should climb the stairs as shown in Figure 5. Because the tread depth is very close to the critical value $(392 \mathrm{~mm})$, the robot will climb the stairs in a critical situation just as shown in Figure 6-(b). The process of robot's stairs climbing is simulated by the software, as shown in Figure 9. The simulation results basically validate the above analysis. The track curves of the five wheel's center are drawn during the climbing process, as shown in Figure 9 and 10. The position of wheel 3 is very close to the center of gravity of the robot in the vertical direction the curve of which reflects the trajectory of robot's center of mass. In Figure 11, the curves of displacement, velocity and acceleration of wheel 3 during the stair-climbing are drawn. When the wheel 2 and wheel 4 climb through the nosings intense vibrations occur. The curves of accelerations of wheel 1, 3 and 4 are shown in Figure 12. Through comparing the curves in Figure 12, the vibration of 
wheel 3 is relatively small, which shows that the robot with $\mathrm{W}$-shaped track suspensions climbs up the stairs very smoothly.

In another simulating, when the riser height is $150 \mathrm{~mm}$ according to equation sets (1) and (2), and the tread depth $d \subset(371,375)$, the robot's first and third track sections can climb the nosing in turn. Because the tread depth $(270 \mathrm{~mm})$ is not in this range $(371,375)$, the robot will climb the stairs just as shown in Figure 6-(a). The process of robot's stairs climbing is simulated by the software, as shown in Figure 13. The simulation results basically validate the above analysis. The track curves of the five wheel's center are drawn during the climbing process, as shown in Figure 13 and 14.
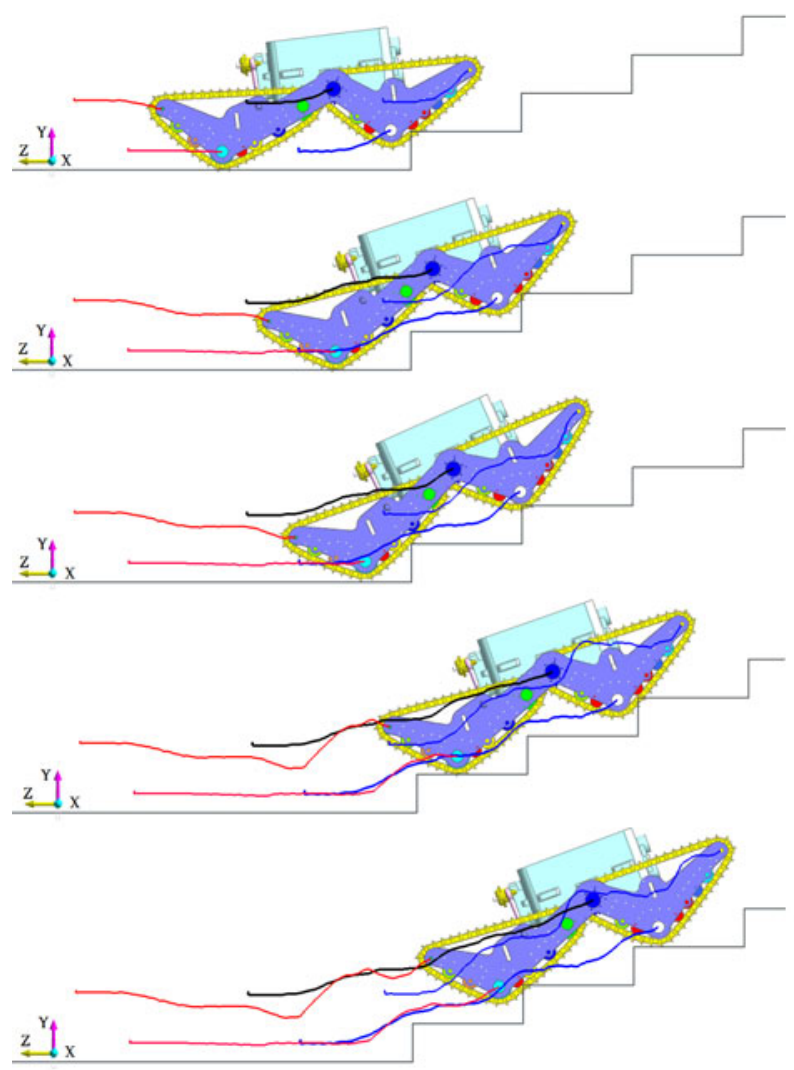

Fig. 9 Stairs-climbing simulation

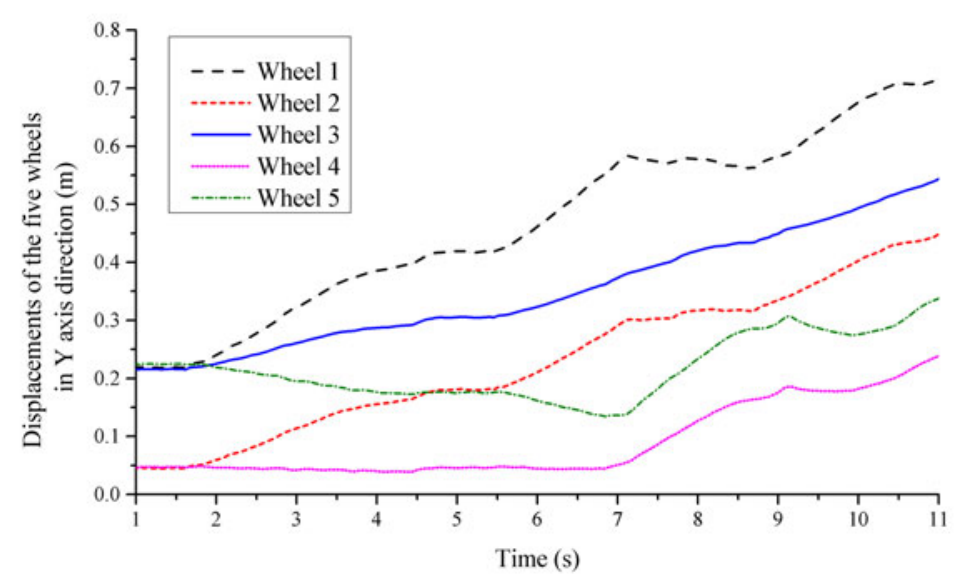

Fig. 10 Displacements of the five wheels in $\mathrm{Y}$ axis direction during the stairs-climbing 


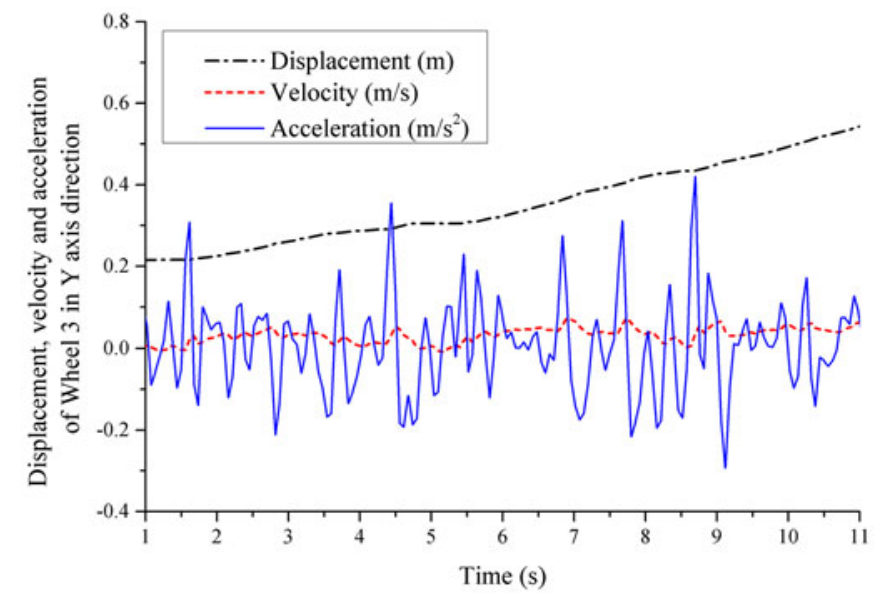

Fig. 11 Displacement, velocity and acceleration of wheel 3 in $\mathrm{Y}$ axis direction during the stairs-climbing

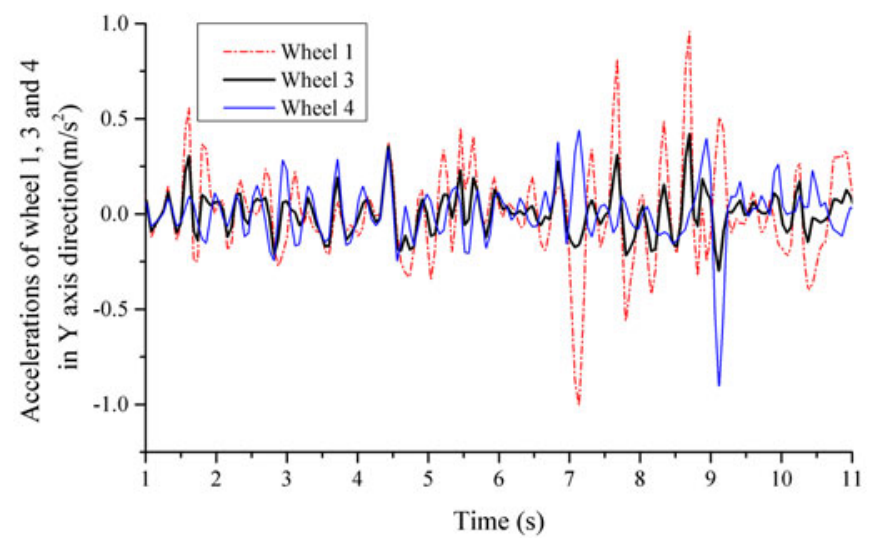

Fig. 12 Accelerations of wheel 1, 3 and 4 in $\mathrm{Y}$ axis direction during the stairs-climbing

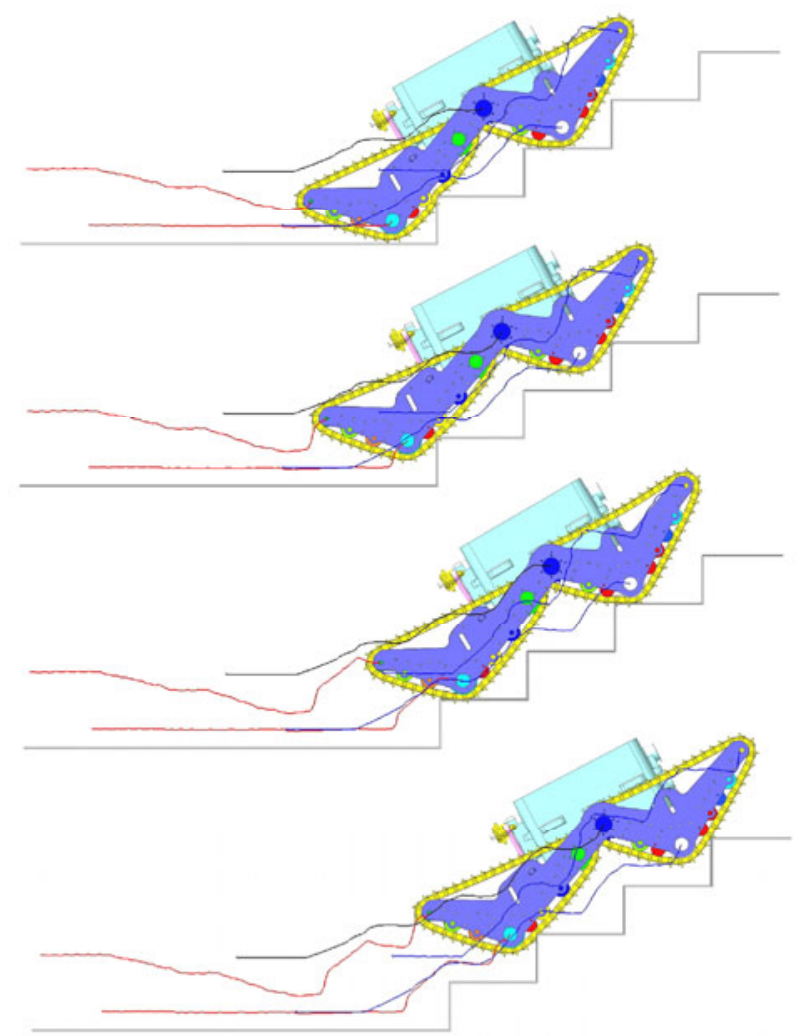

Fig. 13 Stairs -climbing simulation 


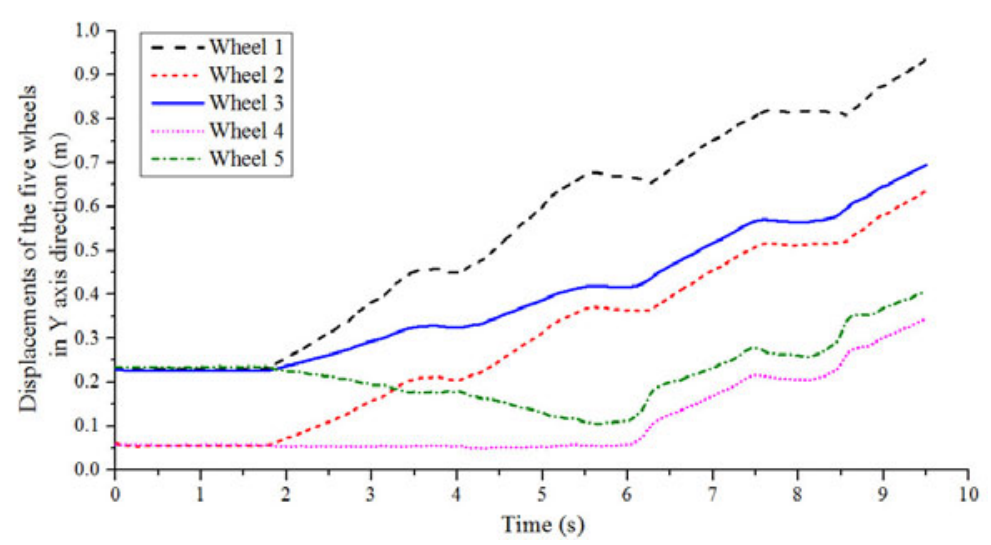

Fig. 14 Displacements of the five wheels in $\mathrm{Y}$ axis direction during the stairs-climbing

\subsection{Continuous stairs-climbing tests}

There are main characteristic parameters of continuous stairs: riser height $h$ and tread depth $d$. Slope $\delta$ of the stairs is calculated by the equation $\delta=\arctan (h / d)$. In a simulated staircase test platform, the parameters of the continuous stairs can be set by adjusting the slope $\delta$ and the riser height $h$. An adjustable parameter simulated staircase test platform is designed to test the stairs-climbing performance of the robot as shown in Figure 15. In this staircase test platform, the slope $\delta$ can be adjusted by a screw rod. The riser height $h$ between each step can also be adjusted by a screw mechanism, so that the platform can be adjusted to the preset parameters. The slopes on both sides of the platform can help the robot climb up or climb down the test platform. Figure 15-(a) is a 3D model of the test platform. Figure 15-(b) is the photo of test platform with a larger slope. Figure 15-(c) is the photo of test platform with a small slope. A large number of climbing tests have been completed on the test platform in laboratory, as shown in Figure 16, and the test results verify the previous theoretical analysis. But some of the test parameters exceed the adjustable range of the test platform and these tests are carried out on the real staircase.

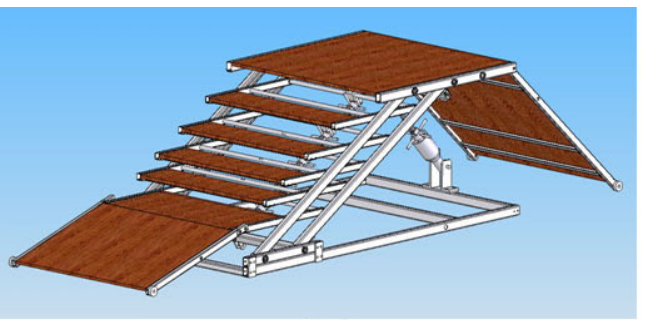

(a)

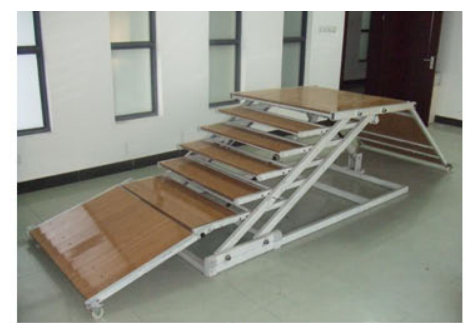

(b)

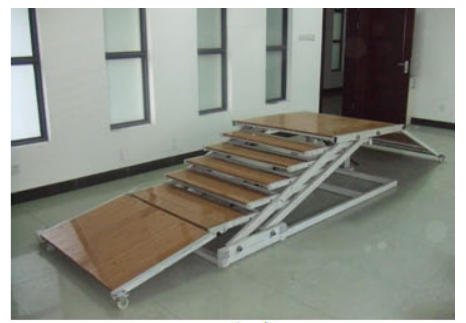

(c)

Fig. 15 An adjustable parameter simulated staircase test platform

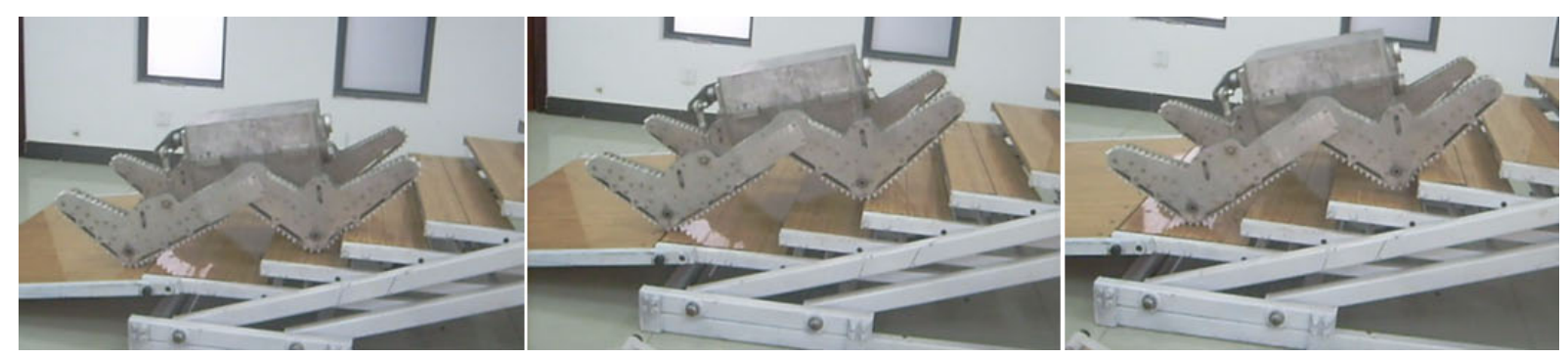

Fig. 16 Stairs-climbing test on the test platform

Some continuous stairs-climbing tests are carried out on the serials stairs outside and inside a building. The riser height and the tread depth of the stairs outside the building are 
$135 \mathrm{~mm}$ and $390 \mathrm{~mm}$ respectively. In this testing process, when the front tracks climb the first step of the stairs, the rear touchdown wheels are moving on the floor (Figure 17-(a)). After the front touchdown wheels climb on the first step's tread, the rear touchdown wheels are still on the floor. Then with all the touchdown wheels on the horizontal plane, the robot moves forward and contact the second step's nosing. At last, the robot begins to climb the second step (Figure 17-(b)). During the climbing process, the rear touchdown wheels always move on the floor. After the front touchdown wheels climb up on the second step's tread, the rear track are ready to climb the first step's nosing, which is just meet the critical situation (Figure 17(c)). When the rear track climb up the first step and rear touchdown wheels are on the tread, the robot has finished the first step's climbing (Figure 17-(d)). The test verifies the theoretical derivation.

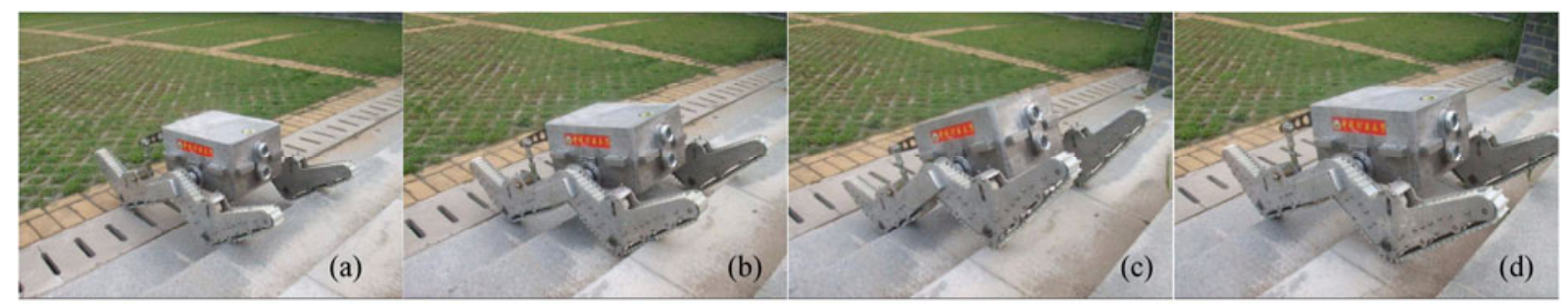

Fig. 17 Stairs-climbing test outside a building

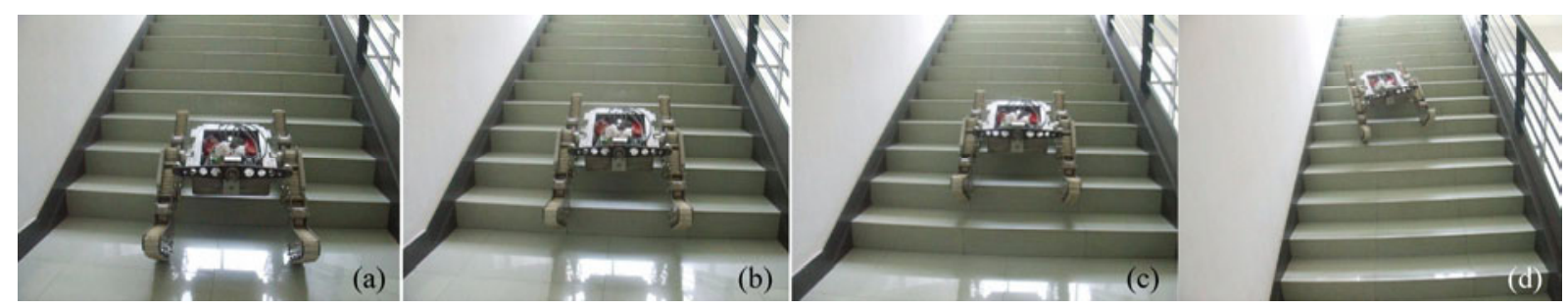

Fig. 18 Stairs-climbing test indoor

The staircase in Figure 18 is a standard indoor staircase, which is paved by the smooth ceramic tile. The tread depth and the riser height are $300 \mathrm{~mm}$ and $140 \mathrm{~mm}$ respectively. The robot's front and rear tracks climb two nosings simultaneously, as shown in Figure 18-(a-c). When the common track robot climbs stairs, the centre line of the robot should be parallel to the slope direction of the stairs. Because the ceramic tile is too smooth, if the robot is not on a good control, the robot may slip or sideslip and must be adjusted its attitude through control. But it is difficult to keep the robot climbing stairs in parallel situation in actual environments. Figure 18-(d) shows the side slipping situation on the stairs.

When the robot climb continuous stairs obliquely, the two track suspensions will swing to adapt to the stairs and the tracks can contact with the nosings and the treads under the action of the differential mechanism. The process and feature of stair climbing obliquely are analyzed in article [9].

\section{Conclusion}

Terrain in underground coal mine after explosion disaster is unstructured. There are artificially built independent steps, continuous stairs, slopes, drainage channels and rails in the laneways. The coal mine rescue robots for detecting after the disaster should have high terrain passing performances. The stairs-climbing capacity of a mobile robot is an important measure of obstacle-navigation performance. This paper introduces a rocker type asymmetrical Wshaped track robot, which has two asymmetrical W-shaped track suspension linked on the both side of the main body. For studying on stairs-climbing capacity of the robot, theoretical analysis, kinematics simulation and tests on real stairs have been carried out. 
In this paper, the two stairs-climbing situations of the $\mathrm{W}$-shaped robot are analyzed. According to the sizes of normal stairs, there are two climbing situations:

- The first situation is that the first track section and the third track section climb up the nosings of the two adjacent steps or two septal steps successively.

- The second situation is that the front and rear tracks climb two nosings simultaneously.

There are two critical states:

- The first and third track section just touch the nosing of two interval steps,

- The first and third track section barely touches the nosings of the adjacent steps.

According to the two critical, equation sets which describe the relationship between tread depth and riser height are obtained. According to the physical dimensions of the robot, the relation curves are drawn corresponding to the equation sets. Conditions of the two climbing situations are obtained. The stairs-climbing are simulated by RecurDyn, and tests on an adjustable parameter simulated staircase test platform in laboratory and real stairs in and outside the building are carried out. The simulation and tests results have verified the results of the theoretical analysis.

This paper can be helpful to optimize the shape and size of the W-shaped track suspension according to the parameters of the stairs, and also guide the application of the robot with a $\mathrm{W}$-shaped track moving mechanisms.

The performance of a robot's stairs-climbing is also related to the dynamic performance and motion control of the robot. This paper is not related to the relevant research which will be studied in future research papers.

\section{Acknowledgements}

This work was financially supported by National Natural Science Foundation of China (No. 51675518), Six Talent Peaks Project in Jiangsu Province (No. JXQC-008), China Scholarship Council (No. 201706425041) and A Project Funded by the Priority Academic Program Development of Jiangsu Higher Education Institutions.

\section{REFERENCES}

[1] Murphy R. R.; Kravitz J.; Stover S. L.; Shoureshi R.; Mobile robots in mine rescue and recovery. IEEE Robotics and Automation Magazine, 2009, 16, 91-103. https://doi.org/10.1109/mra.2009.932521

[2] Gao J. Y.; Gao X. S., Zhu J. G.; Zhu W.; Wei B.Y.; Wang S. L.; Coal mine detect and rescue robot technique research. In: Proceedings of the 2009 IEEE International Conference on Information and Automation. Zhuhai. pp. 1068-1073. https://doi.org/10.1109/icinfa.2009.5205076

[3] Li Y. W.; Ge S. R.; Zhu H.; Mobile platform of rocker-type coal mine rescue robot. Mining Science and Technology (China), 2010, 20 (3), 466-471. https://doi.org/10.1016/s1674-5264(09)60227-1

[4] Li Y. W.; Ge S. R.; Zhu H.; Liu J.; Obstacle-surmounting mechanism and capability of fourtrack robot with two swing arms. Robot (China), 2010, 32 (2), 157-165. https://doi.org/10.3724/sp.j.1218.2010.00157

[5] Li Y. W.; Ge S. R.; Wang X.; Wang H. B.; Steps and stairs-climbing capability analysis of sixtracks robot with four swing arms. Applied Mechanics and Materials, 2013, 397-400 (2013), 1459-1468. https://doi.org/10.4028/www.scientific.net/amm.397-400.1459

[6] Zhao, J.C.; Gao, J.Y.; Zhao, F.Z.; Liu, Y. A search-and-rescue robot system for remotely sensing the underground coal environment. Sensors, 2017, 17 (10), 2426. https://doi.org/10.3390/s17102426 
[7] Li Y. W.; Ge S. R.; Zhu H.; Deduction of the rocker-type track suspension configurations and their applications to coal mine rescue robots, Robot(China) , 2010, 32 (1), 25-33. https://doi.org/10.3724/sp.j.1218.2010.00025

[8] Li Y. W.; Ge S. R.; Zhu H.; Mobile platform of a rocker-type w-shaped track robot. Key Engineering Materials, 2010, 419-420, 609-612. https://doi.org/10.4028/www.scientific.net/kem.419-420.609

[9] Li Y. W.; Ge S. R.; Liu W. L.; Tian F.; Analysis and test on rough terrain adaptation performance of the rocker-type w-shaped track coal mine exploring robot. Information Technology Journal, 2013, 12 (14), 2921-2928. https://doi.org/10.3923/itj.2013.2921.2928

[10] Li, Y.W.; Yan, X.C.; Tian, F.; Zhao, D.L.; Li, B. Analysis and Test on Step Surmounting Performance of a W-shaped Track Robot. The Open Automation Control Systems Journal, 2015, 7 (1), 74-83. https://doi.org/10.2174/1874444301507010072

[11] Liu M. L.; Zhang J.; Li K.; Obstacle performance of track robot with passive rocker based on RecurDyn. Applied Mechanics and Materials, 2013, 397-400, 1580-1588. https://doi.org/10.4028/www.scientific.net/amm.397-400.1580

Submitted: $\quad 27.7 .2017$

Accepted: $\quad 27.3 .2019$
Yunwang Li

School of Mechatronic Engineering, China University of Mining and Technology, Xuzhou 221116, China yunwangli@cumt.edu.cn Sumei Dai $\bowtie$ School of Mechanical and Electrical Engineering, Xuzhou University of Technology, Xuzhou 221018, China sumei-dai@hotmail.com

Feng Tian, School of Mechatronic Engineering, China University of Mining and Technology, Xuzhou 221116, China

Yuwei Zheng

School of Mechatronic Engineering, China University of Mining and Technology, Xuzhou 221116, China

Corresponding Author e-mail: sumei-dai@hotmail.com 